\title{
Fate of the Mach cone in covariant transport theory
}

\author{
Denes Molnar \\ Physics Department, Purdue University, West Lafayette, IN 47907, USA \\ RIKEN BNL Research Center, Brookhaven National Laboratory, Upton, NY 11973, USA
}

\begin{abstract}
An intriguing potential signature of hydrodynamic behavior in relativistic $A+A$ reactions at Relativistic Heavy Ion Collider (RHIC) energies is conical flow induced by fast supersonic particles traversing the hot and dense medium. Here I present first results on the evolution of Mach shocks in $2 \rightarrow 2$ covariant transport theory, in a static uniform medium.
\end{abstract}

Keywords: Mach shocks, covariant transport theory, heavy-ion collisions

PACS: $12.38 . \mathrm{Mh} ; 25.75 .-\mathrm{q} ; 25.75 . \mathrm{Gz}$

\section{INTRODUCTION}

There has been a lot of recent theoretical interest in conical flow in heavy-ion collisions[1, 2, 3, 4]. Important open questions are how the collision dynamics (rapid expansion, phase transition, inhomogeneities) and dissipation (viscosity) affect this unique flow pattern.

It would be natural to investigate these questions using causal dissipative hydrodynamics, however, the $3+1 \mathrm{D}$ codes required still have to be developed. Here I employ a convenient alternative [5], covariant transport theory, which is fully causal and stable, can address full 3+1D, and has a well-defined hydrodynamic limit.

\section{MAIN RESULTS}

I consider, as Refs. [6, 7, 8, 9, 10], Lorentz-covariant on-shell Boltzmann transport theory with elastic $2 \rightarrow 2$ rates, but here couple the system to an external current through the transport equation:

$$
p_{1}^{\mu} \partial_{\mu} f_{1}=S\left(x, \vec{p}_{1}\right)+C[f, f]\left(x, \vec{p}_{1}\right)+C\left[f_{e x t}, f\right]\left(x, \vec{p}_{1}\right)
$$

where

$$
C[f, g] \equiv \iint_{34}\left(f_{3} g_{4}-f_{1} g_{2}\right) W_{12 \rightarrow 34} \delta^{4}\left(p_{1}+p_{2}-p_{3}-p_{4}\right) .
$$

The integrals are shorthands for $\int_{i} \equiv \int d^{3} p_{i} /\left(2 E_{i}\right)$, the source term $S$ generates the initial conditions, while $f_{\text {ext }}$ represents the external current. Because the interest is to study the theory near its hydrodynamic limit, I take $f$ and $f_{e x t}$ to be the phasespace distribution of massless "quasi-particles". This ensures that the equation of state $e=3 p$ is close to that of the high-temperature plasma in the early stages at RHIC. The transition probability 
$W=s\left(s-4 m^{2}\right) d \sigma / d t$ is adjusted to control the shear viscosity $\eta \approx 4 T /\left(5 \sigma_{t r}\right)$, where $\sigma_{t r}$ is the transport cross section.

In this exploratory study I compute the flow pattern generated by an external "jet" moving in the $+z$ direction in a static, uniform, thermal bath (a massless gas of quarks and gluons - 3 flavors, 3 colors) with temperature $T_{0}=0.385 \mathrm{GeV}$ and shear viscosity $\eta \approx 0.075 s$, where $s$ is the entropy density. The corresponding energy density and mean free path are $e_{0} \approx 44.7 \mathrm{GeV} / \mathrm{fm}^{3}$ and $\lambda=0.125 \mathrm{fm}$. Though covariant transport treats interactions between jet and medium self-consistently, I here explicitly turn off jet recoil, similar in spirit to linear response studies. The jet is created at $t=0$.

In the first 'perturbative' scenario the jet deposits energy and momentum through $2 \rightarrow 2$ interactions as encoded in (1). This implies $d E / d L \approx d p_{z} / d L$ at high jet energies $E \gg T$. Typical Debye-screened $t$-channel processes are quite inefficient at energy-momentum transfer to the medium, $d E / d L \sim\left(\mu_{D}^{2} / \lambda_{M F P} T\right) \ln \left(E T / \mu_{D}^{2}\right)$. To maximize effects, I therefore take more optimistic isotropic scattering, for which $d E / d L \sim$ $E /\left(2 \lambda_{M F P}\right)$. The jet is modeled through a moving sharp sphere profile

$$
f_{\text {ext }}^{\text {pert. }}(t, r, z, \vec{p}) \propto \frac{1}{R^{3}} \Theta\left(R^{2}-\left(r^{2}+(z-t)^{2}\right)\right) \delta^{2}\left(\vec{p}_{T}\right) \delta\left(p_{z}-E\right)
$$

of radius $R=0.2 \mathrm{fm}$, where $E$ is the jet energy, and cylindrical $(r, z, \theta)$ coordinates are employed.

Figure 1 shows the jet-induced change in energy density and momentum density in the medium, at $t=2.5 \mathrm{fm}$, for $E=8.25 \mathrm{GeV}$ and $d E / d L \approx d p_{z} / d L \approx 20 \mathrm{GeV} / \mathrm{fm}^{3}$. The largest effect is, of course, right at the position of the jet but there clearly is a bow-shaped front trailing the jet. In the wake of the jet $(z \lesssim 2 \mathrm{fm}, r \lesssim 0.3 \mathrm{fm})$, momentum flows along the jet direction (characteristic "diffusion wake"). These results agree qualitatively with ideal hydrodynamic calculations [4]. This is quite remarkable because in this calculation energy-momentum deposition is not thermal, and there is also a finite, albeit small viscosity.

As an alternative scenario, consider a source that deposits thermalized energy only but no momentum $\left(d p_{z} / d L=0\right)$. This can be incorporated through additional thermal particle production, i.e., adding to the source term $S$ in (1) a contribution

$$
\Delta S(t, r, z, \vec{p}) \propto v \frac{d E}{d L} e^{-\left[r^{2}+(z-v t)^{2}\right] /\left(2 \sigma^{2}\right)} e^{-p / T_{0}}
$$

while putting $f_{\text {ext }}=0$. Here a Gaussian spatial profile was chosen with a width $\sigma=$ $0.3 \mathrm{fm}$, and $v$ is the source velocity.

Figure 2 shows the change in energy density and momentum density in the medium, at $t=2.5 \mathrm{fm}$, in the "pure energy" scenario for $d E / d L \approx 75 \mathrm{GeV} / \mathrm{fm}^{3}$ and $v=0.9 c$. Because the source is turned off for $t>2 \mathrm{fm}$, the bow-shock is now more pronounced. In contrast to the "perturbative" scenario, in the wake region we see momentum flow away from the jet. In the regions with the highest momentum density, the flow angle is consistent with $\approx 50$ degrees expected from the Mach formula $\cos \theta=c_{s} / v$ (here $c_{s}^{2}=1 / 3$ ). These features agree qualitatively with ideal hydrodynamic calculations [4].

For bow shocks to manifest, a low viscosity is crucial. As seen in Fig. 3, the disturbances get largely "washed out" if the shear viscosity is quadrupled to $\eta \approx 0.3 s$. 

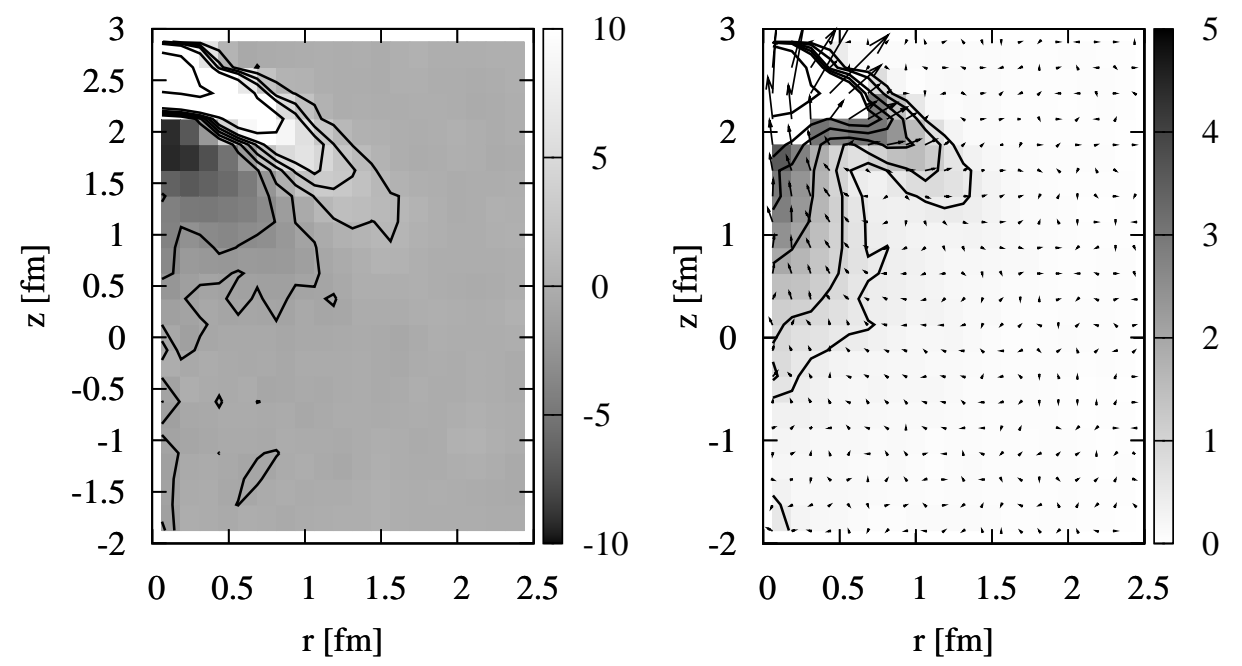

FIGURE 1. Distribution of the local energy density (left) and the magnitude of momentum density perturbations (right), at $t=2.5 \mathrm{fm}$ for the "perturbative" scenario. Energy density contour lines are at -3 , $-1,1,3,10$ and $40 \mathrm{GeV} / \mathrm{fm}^{3}$; while for the momentum density at $0.5,1,2,3,4$, and $20 \mathrm{GeV} / \mathrm{fm}^{3}$. Arrows indicate the local momentum density vectors.
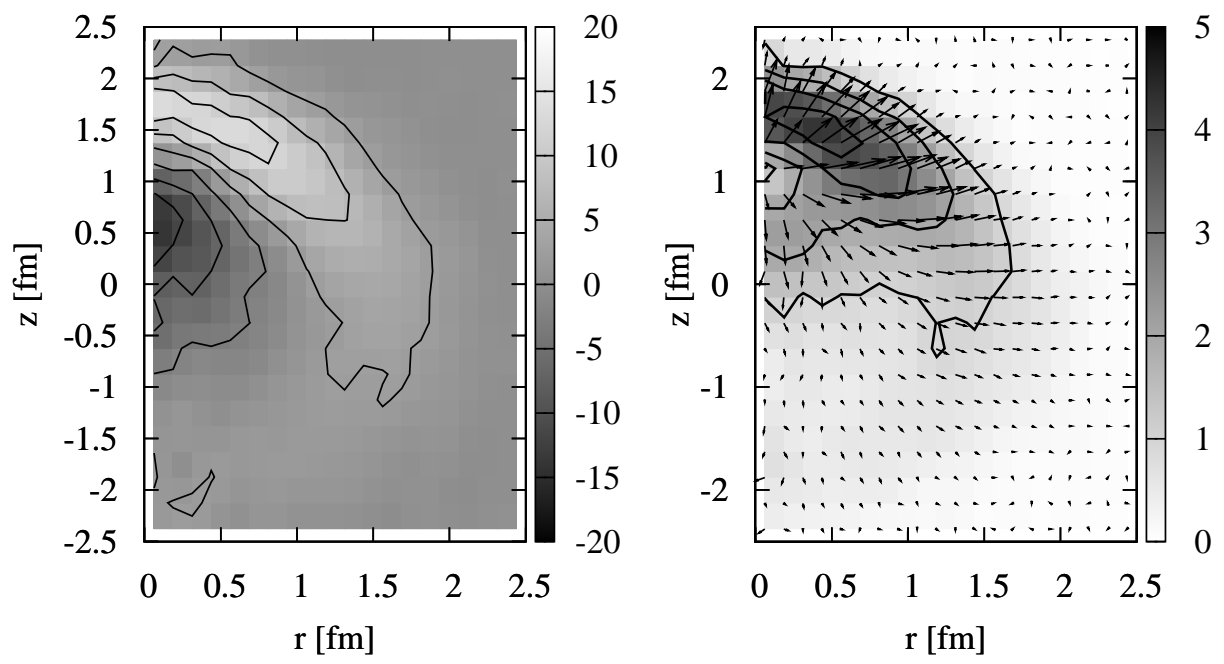

FIGURE 2. Same as Fig. 1 but for the "pure energy" scenario, with contour lines at $-12.5,-7.5,-2.5$, $2.5,7.5$, and $12.5 \mathrm{GeV} / \mathrm{fm}^{3}$ for energy density and $1,2,3$, and $4 \mathrm{GeV} / \mathrm{fm}^{3}$ for momentum density.

For the modest number of test particles and events in this exploratory study, there were no clear signals in the azimuthal distribution in the $x-z$ plane, $d N / d y d \phi$, and the angular distribution relative to the jet axis $d N / d \cos \theta$ (even when weighted by powers of momentum). Ideal hydrodynamic calculations also find very small effects, confined to thermal tails $p / T \sim 20$ [4]. It remains to be seen whether the dynamics and selfconsistent jet-medium coupling can generate appreciable signals in heavy-ion collisions. 

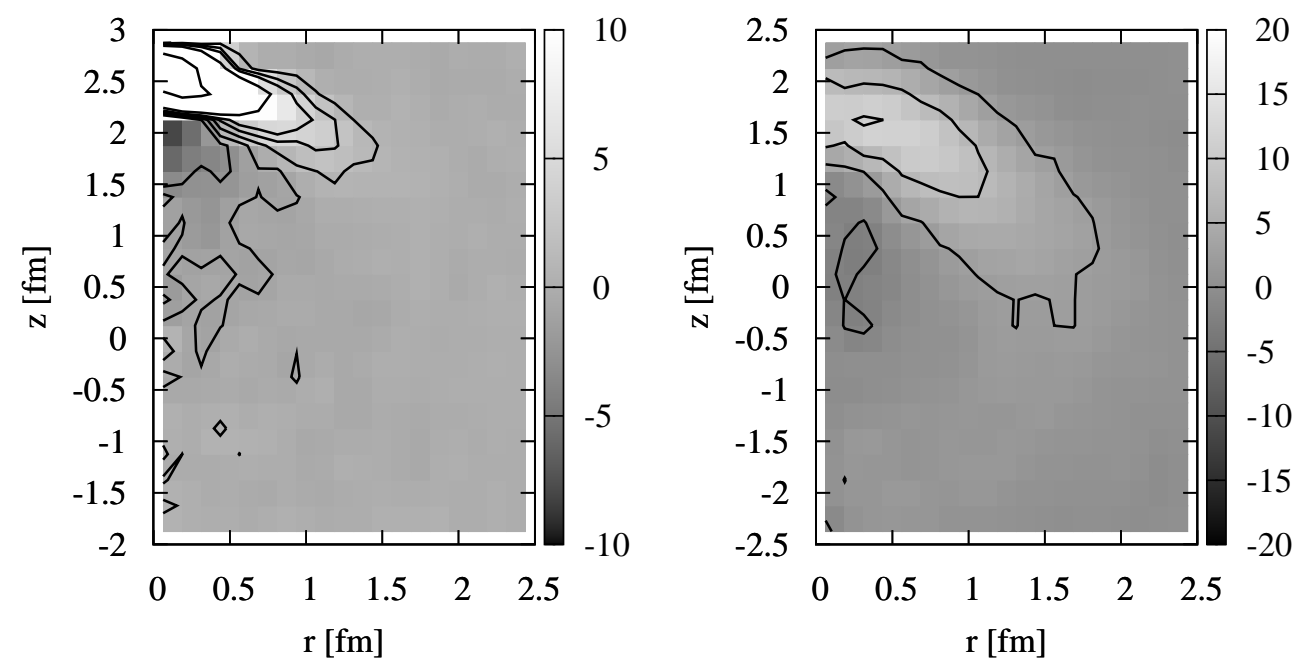

FIGURE 3. Distribution of the local energy density perturbations for the "perturbative" (left) and "pure energy" scenarios (right), same as Figs.11(left) and Figs. 2(left), but with four times larger mean free path.

\section{CONCLUSIONS}

In this work I investigate Mach shocks in $2 \rightarrow 2$ covariant transport theory, in a static uniform medium. If the shear viscosity to entropy density ratio is very low, $\eta / s \approx 0.075$, the results are in qualitative agreement with ideal hydrodynamic calculations[4]. This demonstrates the feasibility of utilizing covariant transport in future 3+1D conical flow studies to incorporate viscosity and a self-consistent coupling between jet and medium.

\section{ACKNOWLEDGMENTS}

I thank RIKEN, Brookhaven National Laboratory and the US Department of Energy [DE-AC02-98CH10886] for providing facilities essential for the completion of this work.

\section{REFERENCES}

1. H. Stoecker, Nucl. Phys. A 750, 121 (2005)

2. J. Casalderrey-Solana, E. V. Shuryak and D. Teaney, J. Phys. Conf. Ser. 27, 22 (2005) [Nucl. Phys. A 774, 577 (2006)]

3. R. B. Neufeld, B. Muller and J. Ruppert, Phys. Rev. C 78, 041901 (2008) [arXiv:0802.2254[hep-ph]].

4. B. Betz et al, Phys. Rev. C 79, 034902 (2009) [arXiv:0812.4401 [nucl-th]].

5. D. Molnar, arXiv:0806.0026 [nucl-th].

6. B. Zhang, Comput. Phys. Commun. 109, 193 (1998).

7. M. Gyulassy, Y. Pang and B. Zhang, Nucl. Phys. A 626, 999 (1997).

8. B. Zhang, M. Gyulassy and C. M. Ko, Phys. Lett. B 455, 45 (1999).

9. D. Molnar and M. Gyulassy, Phys. Rev. C 62, 054907 (2000).

10. D. Molnar and M. Gyulassy, Nucl. Phys. A697, 495 (2002), A703, 893(E) (2002); ibid. A698, 379 (2002). 